\title{
Influence of dual template on the synthesis of size controllable zeolite SAPO-34
}

\author{
Wencan Xiao, ${ }^{1, a}$, Bo Zhang ${ }^{1, b}$, Hao Xu ${ }^{1, c}$, Song Xiao ${ }^{2, d}$, Yanna Liu ${ }^{1, e}$ \\ ${ }^{1}$ Faculty of Chemical Engineering, Kunming University of Science and Technology, Kunming \\ 650500 , China \\ ${ }^{2}$ Faculty of Metallurgical and Energy Engineering, Kunming University of Science and Technology, \\ Kunming 650093, China

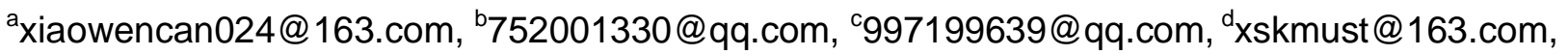 \\ enuo2004lyn@163.com
}

Keywords: Nanoparticles, SAPO-34, template agent, Crystal structure

Abstract. Nanosize SAPO-34 zeolite was successfully prepared by hydrothermal method using tetraethylammonium hydroxide(TEAOH) and triethanolamine(TEA) as template. It was investigated by X-ray diffraction and scanning electron microscopy. The results show that the dual template can control the particle size and change the cr4ystal morphology by adjusting the molar ratio of TEA/TEAOH in the synthesis solution.

\section{Introduction}

SAPO-34 is a member of the SAPO-n series of molecular sieves with successfully synthesized by the Union Carbide Corporation in 1982[1]. This kind of molecular sieve is a new kind of silicon aluminum phosphate molecular sieve, which is formed by $\mathrm{Si}$ substitution mechanism into the aluminum phosphate skeleton sieve. SAPO-34 molecular sieve show very high selectivity in the low carbon olefin, because it has a medium strength acid sites, eight membered ring orifice diameter and high hydrothermal stability[2]. Thus, it is often used to be catalyst in methanol to olefins (MTO) reaction.

The morphology and grain size of SAPO-34 zeolite has an important effect on its performance, therefore, how to control the morphology and grain size of molecular sieve has a high research value. In the hydrothermal synthesis conditions, many factors will affect the morphology and grain size of SAPO-34 molecular sieve, for example template[3], aging time[4], crystallization time[5], raw material ratio and so on. The template has a significant impact on SAPO-34 molecular sieve. Template can induce the formation of molecular sieve crystal in the synthesis of molecular sieve and template plays an important role in structure guided, charge matching and space filling[6]. A number of organic amines such as tetraethylammonium hydroxide (TEAOH), triethanolamine (TEA), diethylamine (DEA), morpholine (MOR) and mixed templates have been used in the synthesis of SAPO - 34. Many researchers have studied the effect of single template on the synthesis of SAPO-34 molecular sieve. In this paper, SAPO-34 samples have been with mixed templates of tetraethylammonium hydroxide (TEAOH) and triethanolamine (TEA) by hydrothermal method.In addition the effects TEA/TEAOH molar ratio on the morphology and grain size of prepared SAPO-34 have been investigated and the properties are analyzed by XRD and SEM. 


\section{${ }^{1}$ Experimental}

In experiment, aluminum isopropoxide, tetraethylorthosilicate (TEOS), phosphoric acid, mixture of TEAOH and TEA are used as aluminum source, silica source, phosphorus source and templates respectively. Zeolites SAPO-34 were prepared via hydrothermal synthesis with a molar composition of $1 \mathrm{Al}_{2} \mathrm{O}_{3}: 2 \mathrm{P}_{2} \mathrm{O}_{5}: 0.6 \mathrm{SiO}_{2}$ :(xTEA:yTEAOH): $115 \mathrm{H}_{2} \mathrm{O}(\mathrm{x}: \mathrm{y}=2: 2,3: 1,4: 0)$. Firstly, TEOS was dissolved with deionized water in a clean polypropylene bottle under vigorous stirring. Mixture of TEAOH and TEA, aluminum isopropoxide and $\mathrm{H}_{3} \mathrm{PO}_{4}$ were slowly added into the above solution with agitation by sepuence. After that, the mixture was transferred into $50 \mathrm{~mL}$ Teflon-lined stainless steel autoclave to age for $48 \mathrm{~h}$ with stirring, which was then crystallized at temperature of $473 \mathrm{~K}$ for $16 \mathrm{~h}$. The as-synthesized sample was obtained by centrifugation, washed and dried at $105^{\circ} \mathrm{C}$ overnight. At last, calcination was carried out in air at $550^{\circ} \mathrm{C}$ for $5 \mathrm{~h}$ to remove the template agent molecules. The as-synthesized samples were labeled as a, b, c, where a, b, c represented the molar ratio of 2:2, 3:1 and 4:0 respectively..

X-ray diffraction (XRD) patterns were recorded on a Rigaku D/max-2200 X-ray diffractometer by using a $\mathrm{Cu}-\mathrm{K} \alpha$ radiation operating at $40 \mathrm{kV}$ and $40 \mathrm{~mA}$ in and a $\mathrm{Ni}$ filter in the range of $2 \theta=5-50^{\circ}$. Scanning electron microscopy (SEM) was performed using hitachi S-4800 3.0kV microscope.

\section{Results and discussion}

Fig. 1 shows the XRD patterns of samples prepared with different molar ratio of TEA/TEAOH.A detailed examination of the diffraction pattems reveals the formation of a SAPO-34 rhombohedral structure. The high crystallinity of the samples are indicated by the single peaks around $2 \theta$ degree at $9.5^{\circ}, 12.8^{\circ}, 16.0^{\circ}, 18.8^{\circ}, 20.6^{\circ}$ and the double peaks at $26^{\circ}, 31^{\circ}[7,8,9]$. The peak intensities are observed to differ based on the molar ratio of TEA/TEAOH, indicating that the extent of crystallization and the size of the crystallites on the SAPO-34 are dependent on the molar ratio of TEAOH/TEA. The sample $a$ and $b$ have the same characteristic peak as the standard SAPO-34, indicating that the synthesized product are SAPO-34. The characteristic peak intensity of sample $b$ is higher than that of sample $a$, which illustrates that the crystallinity of sample $b$ is relatively high. The overlapped double peak of sample a are more than that of sample b, which also can be proved that the relative crystallinity of sample $b$ is higher than that of sample a. There are SAPO-34, SAPO-5 and SAPO-11 characteristic peaks in sample c, explaining the synthesized product contain SAPO-34, SAPO-5 and SAPO-11 molecular sieve. Low $\mathrm{pH}$ is conducive to form AFI structure of the molecular sieve in the process of SAPO molecular sieve to form crystal. When the $\mathrm{pH}$ is increased, the CHA and AFI structure competition tends to form a CHA structure. It shows that weak acid is helpful to generate SAPO-5 of AFI structure[10,11]. At the same time, SAPO-11 is formed under acidic condition. Therefore, SAPO-5 and SAPO-11 are prone form with increase of the amount of TEA. That ration of TEA/TEAOH will affect the structure of molecular sieve, and the unpurified molecular sieve is obtained when the molar ratio of TEA/TEAOH is more than a certain proportion. 


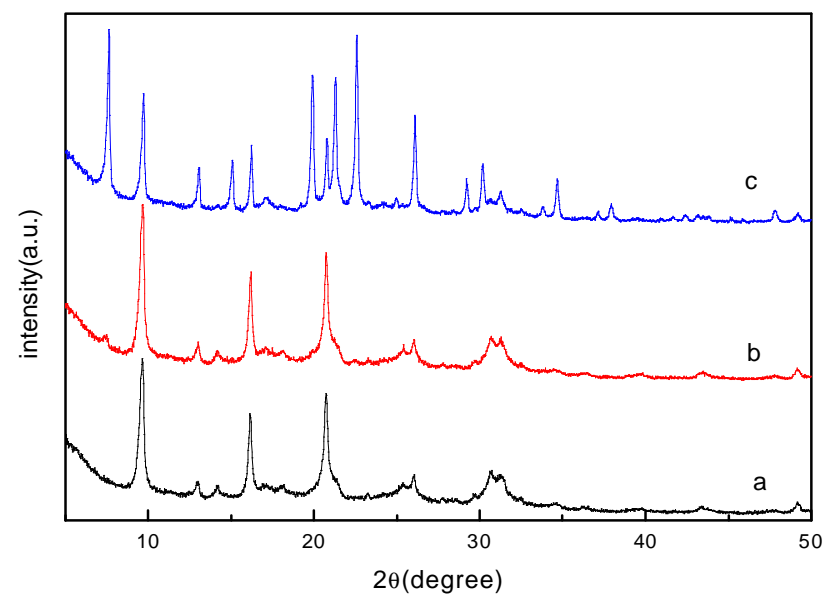

Fig.1. XRD patterns of SAPO-34 samples with TEA/TEAOH. a, b, c represented the molar ratio of $2: 2,3: 1$ and 4:0 respectively.

The morphology of prepared samples has been determined by SEM in Fig.2. From the images, it depicts that the amount of template employed in the preparation affects the properties of the samples. When a small molar ratio of TEA/TEAOH $(\mathrm{x}: \mathrm{y}=2: 2)$ is used, most of the crystals are spherical and the surface is rough, and crystals' size is about $1.5-2 \mu \mathrm{m}$. At the same time, there are some amorphous substance in picture A, which it is consistent with the low intensity of characteristic peaks in XRD of samples a. With the growth of template molar ratio(TEA/TEAOH) to $\mathrm{x}: \mathrm{y}=3: 1$, SAPO-34 particles grow bigger and bigger. But the crystal surface becomes smoother and smoother, revealing that the relative crystallinity of the crystal is higher. When the molar ratio of (TEA/TEAOH) is used with 4:0, the crystal morphology and crystal size distribution of the samples are not regular, because there is SAPO-5 and SAPO-11 formation in sample c. The SEM results prove the correctness of the results of XRD spectra.
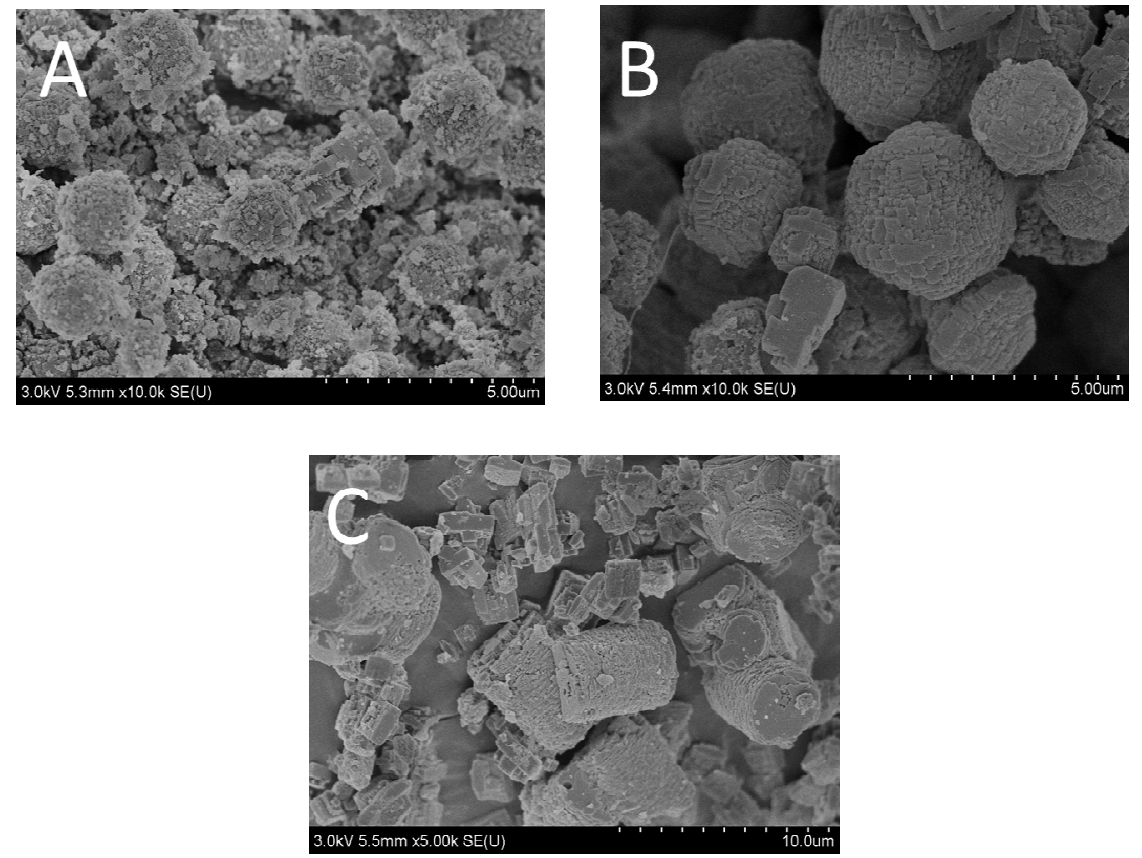

Fig.2. SEM images of SAPO-34 samples with TEA/TEAOH. a, b, c represented the molar ratio of $2: 2,3: 1$ and 4:0 respectively. 


\section{Conclusions}

Nano-scale SAPO-34 molecular sieve has been successfully prepared by hydrothermal method with the addition of dual template. The particle size of obtained SAPO-34 can be controlled by the molar ratio of TEA/TEAOH added in the initial solution and the mixture template molar ratio can affect the morphology of the crystal. We can adjust the size and crystal appearance of SAPO-34 by controlling. This method can be extended to change the morphology of other molecular sieves.

\section{Acknowledgements}

This work is supported by National Natural Science Foundation of China (21301079). Analysis and testing foundation of Kunming University of Science and Technology (20150376, 20150815).

\section{References}

[1] H. X. Liu, Z. K. Xie: Chinese Journal of Chemical Physics. Vol. 16(2004), p. 521

[2] X. S. Chen, Y. Liu, L. Shi: Petroleum Refinery Engineering. Vol. 35(2005), p. 5

[3] Vistad Ø. B, Akporiaye D. E, Taulelle. F: Chemistry of Materials. Vol. 15(2003), p. 1650

[4] H. Dong, S. H. Liu, Y. W. Tang: Journal of Dalian University of Technology. Vol. 3(2014), p. 200

[5] S. Askari, R. Halladj, M. Sohrabi: Reviews on Advanced Materials Science. Vol. 32(2012), p. 83

[6] H. O. Pastore, S. Coluccia, L. Marchese: Annual Review of Materials Science. Vol. 35(2005), p. 351

[7] B. M. Lok, C. A. Messing, P. R. Lyle: Crystalline Silicoaluminophosphates, U. S. Patent, 4,440,871. (1984).

[8] J. Tan, Z. Liu, X. Bao: Microporous and Mesoporous Materials. Vol. 53(2002), p. 97

[9] M. Popova, C. Minchev, V. Kanazirev: Applied Catalysis A: General.Vol. 169(1998), p. 227

[10] Sastre. G, Lewis. D. W, Catlow. C. R. A: Journal of Molecular Catalysis A: Chemical. Vol. 1191997, p. 349

[11] H. J. Sun, J. S. Chang, S. H. Jin: Microporous and Mesoporous Materials. Vol. 64(2003), p. 33 